\title{
Building Governance Capability in Online Communities: A Social Network Perspective
}

\author{
Mohammed Harysi \\ The University of Warwick \\ m.harysi@warwick.ac.uk
}

\author{
Bogdan Negoita \\ HEC Montréal \\ bogdan.negoita@hec.ca
}

\author{
Joe Nandhakumar \\ The University of Warwick \\ joe.nandhakumar@wbs.ac.uk
}

\begin{abstract}
Online communities are increasingly seen as new forms of organising. However, we have limited understanding of how governance emerges in an online community. Prior literature either focuses on governance as a dynamic process-oriented view or as static comparative analysis, in contexts where the online community is mature and well established. This paper therefore seeks to explore how governance evolves throughout the history of an online community, from an embryonic stage, through the emergence stage to the establishe stage. In the context of an online community built around a GitHub-hosted project called GitPoint, we draw on the concept of capability to carry out a theoretical narrative of interactions between individual members that are conducted across social networks, including Twitter and Gitter. Based on this narrative, the paper offers insights into the emergence of governance in an online community and makes key contributions to the literature on governance in such communities.
\end{abstract}

\section{Introduction}

Online communities experience governance challenges unlike those found in conventional hierarchical authority structures and utilise different governance mechanisms [24]. They are open virtual spaces for people with common interests to share and co-create knowledge [34]. These online venues are increasingly seen as new forms of organising that can succeed in creating value, such as in production-based communities [22, 26, 33]. With characteristics such as high turnover, fluid boundaries, sharing of common resources and expertise-based control, these new forms of organising appear to be "governed significantly differently than conventional hierarchical designs" [24:142]. Their shared goals range from software development [27, 37] and healthcare support [11] to serving as sources of innovation for organisations looking outside their boundaries for creative ideas [12]. They enable and enhance networking among a distributed set of participants, where barriers to entering and exiting the community are relatively low. More importantly, one of the most significant problems in online communities concerns how complex social collectives govern, organise and coordinate the actions of geographically dispersed individuals to achieve collective outcomes $[3,33]$.

Today, thousands of geographically dispersed individuals can work together and deliver consistent online output without "the price or corporate system governing the activity" [3:1649]. Their success in such communities depends somewhat on both "task and relational dimensions of their discussions", therefore, establishing a common understanding of the rules that "should govern group members' behaviours can be key to the success of an online group" [9:596]. This form of governance is especially relevant when large numbers of geographically dispersed individuals interact in support of an internet-mediated activity towards specific outputs [15]. This phenomenon has been described as social production, i.e. an alternative form of production organisation facilitated by modern digital technology [6]. Accordingly, social production is understood as an activity that is directed towards creating specific outputs in the absence of governance mechanisms (e.g. managerial structure for coordination) [6]. Linux and many other projects successfully gather the contributions that create significant economic and relational value. Online social production generates innovative solutions at a remarkably low cost of communication and cooperation through new ways of organising. Online communities have been recognised as enablers of social production, as they foster peer-based collaboration and have become sources of innovation $[1,14]$. Yet, an organisational economics perspective 
of social production systems leads only to a limited view of the phenomenon [3].

Despite the advantages that large-scale social production systems may have, they cannot avoid governance problems, i.e. social production systems involve differential interests and information asymmetries [3]. Further, there is a lack of an accepted definition, which leaves it up to each researcher to decide what governance means in communities [30]. Governance has been defined as the means of directing, controlling and coordinating autonomous individuals on behalf of an open source software (OSS) development project [28]. Moreover, it has been contend as an evolving phenomenon in online social production, which "rests on the progressive development of a collective capability to integrate highly distributed knowledge resources and direct them to the joint production of value" [3:1650].

Indeed, much of what we know about governance problems and mechanisms in communities is based on studies of OSS (e.g. [30, 33]). For instance, Shah [35] investigates how differences in governance in OSS and gate-source communities dramatically affect individuals' reasons for participation in such communities. Also, he argues that with growth in the diversity of sponsors of open source projects, new types of governance may emerge. Further, due to the growing elasticity of the open source frame, the community-managed governance model has become decoupled from the notion of an open source project [32]. Markus [30:159] presents a qualitative review and synthesis of the literature on OSS governance, and he argues that it could include the following: (1) both structures and process, (2) informal and formal rules, (3) "externally applied as well as internalised rules" and (4) "mechanisms of both trust and verification/control". Recently, Shaikh and Henfridsson [37], argue that the nature of governance varies across online communities, and it offers the authoritative framework for coordinating activities in open source communities. They also argue that multiple traces of authority may co-exist in its evolution. Such multiplicity has been investigated by examining the authoritative structures that are embedded in coordination processes. Because governance varies across online communities, Wikipedia has been used as a paradigm example to investigate how a form of governance makes online social production possible [3]. They draw on the concept of capability and routine to develop a dynamic, process-oriented view, which departs from the past research focused on static comparative analysis.

As it is stated above, though several studies have enriched our understanding of the evolving governance of online social production in mature online communities [3, 30, 33, 35, 37], much less is known about how governance mechanisms evolve before, during and after the existence of these communities. There are many implications for such limitations of the existing literature. The conclusions based on the partially structural perspective used in prior studies do not reveal the full dynamics of the elements that constitute governance mechanisms of social production in an online community, such as the interactions that occur during its birth and the emergence phase [17]. In short, the literature exhibits a broad variety of views on what constitutes governance in mature online communities. For instance, in some literature, governance has been portrayed as a unified phenomenon, while in other literature, the concept of governance has been operationalised as many different phenomena, such as role structures and technical and managerial processes [30]. This points to a possible reason for the existence of this conceptual issue governance should be conceptualised as a unitary phenomenon [21], or it should be conceptualised as composed of dimensions with diverse manifestations [19]. This diversity of perspectives on governance may rest with when in the lifetime of an online community, studies have focused on governance [17]. Also, previous research investigated governance in contexts where it was embedded to a considerable degree in the technological platform that absorbs much of the organising that goes on in the production system. Technology, i.e. platforms, therefore, is not simply an instrument enabling social production, but a way to govern participation (e.g. peer reviews) [3, 30]. Thus, despite the flexible structures in online communities or Wikipedia, contributors still need to meet some requirements for their work to be valued (e.g. voting software). Online communities have characteristics that cannot be shared with other forms of organising [24]. By contrast, to exchange opinions and share ideas about collaborative projects across social networks (e.g. Twitter), participants do not need to comply with specific requirements, such as written policies and version control software. In the literature, it has been argued that governance is primary informal (e.g. enacted through shared norms), formally documented (e.g. constitutions), or encoded in technology (e.g. version/release control) [30]. It is within this context that path dependency is important in understanding and accounting for how governance typologies change throughout the history of an online community (e.g. during the embryonic, emergence and establish stages).

We have limited knowledge as to whether social production in other domains, such as distributed interaction across social networks prior to the existence of an online community (Twitter and Gitter), face governance challenges other than what we know from 
OSS communities [24]. Thus, while considerable progress has been made in understanding governance in the context of a mature online community, the focus of the extant literature has largely focused on its changing nature over time and how its nature varies across open source communities [37]. We lack satisfactory answers on how a vast and distributed interaction between members of an online community (i.e. GitPoint users) across social networks (Twitter and Gitter) can maintain itself and accomplish concerted performance, as its community comes into existence [3]. To develop a full dynamic view of governance, we adopt a capability based perspective $[3,39]$. We theorise that the evolving governance in online social production $[3: 1650,24,37]$ is rooted in interactions between select individuals across social networks, and it "rests on the progressive development of a collective capability to integrate highly distributed knowledge resources and direct them to the joint production of value".

Specifically based on organisational learning theories, dynamic capabilities emerge from the accumulation of experience in performing organisation routines [40]; therefore, we define a capability as what an individual or another type of collective arrangement can actually do. Following Aaltonen and Lanzara [3:1650], we consider collective governance capability as "the capability of a collective arrangement to steer a production process and an associated interaction system". We claim that a more thorough understanding of continuing interaction across a social network is critical in assessing the evolution and emergence of governance mechanisms in online communities. Hence, the aim of this paper is to address the following research question: How does collective governance capability emerge and evolve in online communities?

To answer the question empirically, we carried out an extensive content analysis of digital trace data [8] of interactions between members of an online community (i.e. GitPoint users) across social networks (Twitter and Gitter), as their online community was coming into existence. Leveraging the research lens of distributed collective governance capability [3, 24, 30, 32, 33], we sought to understand the emergence of governance in the context of their online community.

\section{Theoretical framework}

The emergence of new organisational forms in the context of networked technologies has attracted much attention in the past decades. Attention has concentrated on moving away from the conventional bureaucratic form and has focused on terms like postmodern organisations [10]. According to Bartlett and Ghoshal [5:345], this move means organising so as to "treat people more as assets to be developed than as costs to be controlled, and as renewable strategic resources rather than as replaceable operating parts". Correspondingly, governance problems occur when information relevant to value production is distributed between different actors [18]. Although Bartlett and Ghoshal [5] mainly focused on new organisational forms within firms, and Hayek [18] focused on knowledge exchange governance, the view equally applies to online social production. Summing up, explanatory research is needed to investigate how highly distributed interaction and knowledge across networks (Twitter and Gitter) can be integrated and steered towards a coherent collective output in the online community, through examining the dynamic capabilities of users' interactions prior to the existence of the online community (GitPoint; [3, 15]). Organisationally, online social production systems combine three core characteristics: "(a) decentralization of conception and execution of problems and solutions, (b) harnessing diverse motivations, and (c) separation of governance and management from property and contract" [7:265].

\subsection{Governance in online social production}

A considerable base of scholarship on online social production governance now exists, including free and OSS Wikipedia and production-based communities [3, $33,37]$. Online social production is considered the most radical organisational innovation emerging from internet-mediated social interaction [7]. Writers on OSS governance have rarely defined governance precisely [28], and it can include, for example, empirical research on structures of roles and responsibilities [31], decisions taken by project leaders for major changes [36] and norms of reciprocity [35]. The unique characteristics of online social production make governance a distinct problem that differs from that of markets or traditional hierarchies in terms of creating value [15]. For instance, collaborative relationships among community participants can take place without the structural mechanisms traditionally associated with knowledge collaboration in organisations. Production-based communities may partially overlap with traditional organisational structures, but they are clearly distinguishable from markets or traditional organisational structures (e.g. hierarchies, with regard to creating value). Compared to traditional forms of organising, there are no employment contracts, formal roles or organisational hierarchies to govern individuals' expected knowledgesharing and creation [15]. Basic conditions for hierarchical organisations' (e.g. firms') governance mechanisms and structures simply do not exist [1]. 
Though many online social production systems have developed effective governance mechanisms that enable the collection and integration of knowledge, mobilising and steering a handful of geographically dispersed individuals around a creative idea poses different problems to directing large numbers of people working on a mature product. According to Aaltonen and Lanzara [3], current governance theory tends to rely on a static perspective of governance anchored to discrete institutional forms, failing to capture a dynamic process-oriented view of governance mechanisms in social production. We argue that a deeper understanding of the emergence and evolution of governance in online social production requires reviewing and synthesising the growing body of organisational research and theorising that governance is the capability to progressively "design and implement mechanisms to control and coordinate joint production" [3:1650].

\subsection{Collective governance capability}

Complex and interdependent tasks are accomplished in firms by integrating knowledge into organisational capabilities [20]. Therefore, capabilities embody the knowledge of how to do something, and they enable organisations and individuals to accomplish tasks and activities efficiently [13]. Recent debates on the theoretical convergence of capabilities have acknowledged governance as a distinct capability that can be learned and developed through integrating knowledge, and it is therefore an evolving asset [4, 20]. Accordingly, once a capability is developed and consolidated as an asset, "it may become itself an objective of governance" [2:1652]. Thus, a governance capability may include the governance of other capabilities. In short, knowledge integrated into a governance capability can be perceived as knowledge on how to control and coordinate a distributed system (e.g. motivating people on Twitter to participate in \#GitPoint).

The notions of conceptualised organised production and task-oriented coordination as expressions of knowledge-based organisational capabilities were initially proposed within the knowledge-based view of the firm, but it is reasonable to assume that capabilities "emerge in other types of collective arrangements as well" [2:1652, 16, 29]. Technology has changed the nature of communications and allowed geographically dispersed individuals to share, discuss and build creative ideas with each other (e.g. writing code for an app or open tasks). In addition to facilitation exchange without the mediation of firm hierarchies or markets, this new form of organising also has a generative capacity that "seems to us more crucial in explaining online social production" [1:5]. An online production system is collective in nature; it has the capacity to support the distributed and unsynchronised development of ideas, where the resources used to support such ideas are not found "in a single individual, but are distributed among many individuals" [26:2].

Thus, we stipulate that online social production systems embody a collective set of unique capabilities for doing things such as sharing knowledge and creating entirely new insights and ideas, and it is difficult for firms to strike the right balance between various incentives that motivate individuals and creating and maintaining common-based knowledge resources [15].

\section{Method}

\subsection{Research design}

In this paper, we apply the capability theory perspective to investigate the evolving governance of online social production in online communities, by examining the dynamic properties of users' interactions prior to the existence of the online community (GitPoint). Based on previous studies and our empirical work, we develop a theoretical narrative that focuses on understanding the evolution of governance throughout the history of GitPoint's repository $[3,25]$. A public repository on the GitHub platform (GitPoint https://gitpoint.co) was created on 26 March 2017. The idea of GitPoint was to build an open source application that allows GitHub's users to manage their projects through smartphones. GitPoint has 142 members and is self-managed in allocating tasks, fixing bugs and maintaining codes. Twitter was used by the original owner to announce, promote and screen ideas for the GitPoint repository. In particular, the original owner encouraged participation and embraced views about GitPoint's repository via Twitter. The GitPoint repository was selected for investigating the evolving governance of online social production from distributed interactions across social networks (Twitter and Gitter) for three reasons. Firstl, GitPoint is seen as an open virtual space for social aggregation, where technology enables people with common interests to share and co-create knowledge. Second, GitPoint emerged without traditional organisational structures. Third, GitPoint complies with Kim's [23] proposal regarding successful online communities: a clear vision, flexible spaces, individual roles, leadership and events. 


\subsection{Data collection}

The empirical aspect of this paper required three stages of data collection, which relied primarily on digital trace data capturing and analysis. In the first stage, GitPoint's digital data were collected from GitHub through its automated API functionality. The data included the profiles and exchanges of all users. It included 77 contributors, 65 users, 732 open and closed issues, 358 forks, 416 pull requests and 594 commits. A contributor on GitHub is someone from the outside not on the core development team of the project that wants to contribute some changes to the repository's original files. Users are members of the repository who participate, but their participation has not been approved for inclusion in the original files. GitHub issues are used to track ideas, enhancements, tasks or bugs. The issue file includes GitPoint members' exchanges (e.g., software bugs reports and users' feedback). Forking was another important aspect of our data collection. A fork is a copy of a repository. Forking a repository gives anyone the ability to experiment with changes without affecting the original repository's files. In addition, pull requests on GitHub enable users to inform each other about the changes they have pushed to a branch in a repository. Once a pull request is opened, potential changes can be discussed. However, only users who have the authority can accept and merge changes into the base branch of a repository. A commit, or revision, represents any individual changes that have been added to the files of GitPoint's repository. This file contains commit messages, which are brief clarifications of the changes that have been made.

Indeed, the Twitter accounts of GitPoint users were an important attribute of the data in the first stage of the data collection. These accounts constituted the corpus of the Twitter data that were related to the production-based online community (GitPoint). Having identified Twitter accounts for GitPoint's participants, we were able to retrieve and collect their public microblog posts using Twitter's premium API (i.e. fullarchive endpoint) in the second stage of our data collection. Twitter's premium API provided functionality beyond what is available in the standard search/tweets endpoint. It provided access to the full history of Twitter data since March 2006. We traced all the GitPoint user accounts that we identified in the first stage of the data collection on GitHub by using a Python wrapper for the Twitter API. While this dataset of tweets, retweets and @ mentions contained an extensive amount of data, we concentrated on tweets that were generated prior to the existence of the online community (GitPoint). Yet, even after we sorted the Twitter data for a specific period, an unrelated set of tweets existed (e.g. tweets that were not related to GitPoint's repository). We sought to isolate the unrelated data through reading the entire dataset of posts to identify the tweets, retweets, @ mentions, links and \#hashtags that were related to GitPoint [38].

Further, we recognised Gitter as a communication platform that was used in addition to Twitter. Gitter is a chat and networking platform that helps to manage, grow and connect communities through messaging, content and discovery. Gitter was therefore the third stage of the data collection. The main reason for including Gitter was to triangulate the data points from different sources and to create as complete a story as possible regarding the evolving governance in the GitPoint repository.

\section{The Evolution of GitPoint Governance}

In this section, our approach assumes that the development of GitPoint is associated with the building of a collective capability that its users expressed across social networks (Twitter and Gitter) prior to the existence of the community (GitPoint). Building these collective capabilities was distributed among many individuals as more and more people engaged in conversations and discussions (e.g. tweets and retweets) regarding GitPoint's development. Based on our data analysis, we divided GitPoint evolution into three stages: the embryonic, the emergence and the established stages. The embryonic stage represents the stage prior to the existence of GitPoint (i.e. interactions on Twitter), which we identified as the period from 11 July 2017 to 31 August 2017. At this stage, all the exchanges between GitPoint's users were via Twitter. At the start of GitPoint, there was no managerial capacity, resources exist widely distributed in tweets, and their pattern of distribution is unknown at the beginning.

To put it simply, there was not even an established community for the GitPoint repository; that was yet to take shape. After the owner explicitly announced GitPoint via Twitter: “@hdjirdeh: Couldn't find a @ github iOS app that had everything I needed, so I built one GitPoint - made with @ reactnative; and one welcoming everyone to participate, regardless of their experience", the announcement gained momentum through retweets and tweets by others. The original owner invited everyone to discuss and share their opinions regardless of their experience (e.g. “@hdjirdeh: Don't care whether you have O experience in dev or are an expert in @ reactnative, everybody is always welcome to learn"). At this stage, governance capability was mostly assured by the original owner and enacted through the interaction 
between individuals. The original owner was probably more concerned about how to attract others to participate and about enhancing connectivity and sociability between them, rather than about managing operational software development processes (e.g. managing code quality). In this stage, the governance capabilities that the original owner expressed were mostly promoting GitPoint and attracting others' attention to participate. The rapid expansion, articulation and circulation of content was essential, while less attention was given to coordination work. The findings at this stage align with what Markus [30:156] has called OSS project climate, which is a good project climate engendered by the idea that "democratic governance might actually be more effective at motivating contributions".

The emergence stage is the stage between the embryonic and the established stages. We considered the first two months after the embryonic stage (01 September 2017 to 31 October 2017) as the emergence stage of GitPoint for three reasons. The first reason was due to the increase in the number of participants. The second reason was due to the change in participation (posts) as the number of posts grew increasingly at both the member and the stage levels. Further, the total participation was 1,159 posts; we observed that this increase in the total participation had developed a set of coordinating guides and structures for individual contributions. During this stage, individuals learned to deal with each other, as they gradually externalised their knowledge into rules and guidelines (e.g. “@andrewda: What do y'all think about adding some detox tests? \#575”). Thus, collective capabilities' development engendered the configuration of tasks and groupings of contributions during this stage. These new forms of organising differ from markets or traditional hierarchies in creating value; they make online social production a more effective learning system. Most importantly, we noticed that user participation was focused on technical issues (e.g. writing codes) rather than promoting and encouraging participation during the embryonic stage. We observed that the evolving governance in this stage was more concerned with managing abilities and expertise, contrary to the purpose of governance in the embryonic stage (promoting GitPoint). Experts in this stage engaged in joint work (e.g. fixing bugs), but at the same time, they benefitted from learning how to organise distributed efforts of collective knowledge among community participants. The cost of building collective capability at this stage is not limited to the contribution of the content itself or the cost of communications. The ability to pool distributed knowledge, resources and coordinate action towards shared goals, with the absence of firm hierarchies, is a non-monetary cost [7]. During this stage, we notice that the purpose of governance has changed from the embryonic stage. In the embryonic stage, the purpose of governance is finding solutions to social dilemmas about individuals tweeting about GitPoint. While the purpose of governance in this stage is solving coordination problems during GitPoint's development.

The established stage represents the maturity of GitPoint's repository, wherein governance was fully developed. In this stage, collectively produced rules (user interactions) from the emergence stage seem to have become increasingly formalised and embedded within the internal governance of GitHub's platform. This aligns with what Markus [30] found, in that governance could include informal, formal and encoded rules. Further, newcomers became socialised into an increasingly mature community (GitHub), with demising opportunities to explore or innovate with the dynamics of social production (e.g. participating in the early stages of GitPoint's lifecycle; [3], while incumbent users (users who participated throughout the history of GitPoint) derived value from their established positions in early stages. Therefore, new capabilities had to be developed to maintain, protect and enhance this value over time. The focus changed from collectively developing effective rules to efficiently enforcing them.

\section{Discussion of conceptual development}

In addressing our key research question on how collective governance capabilities emerge and evolve in online communities, we studied a well-established online community and explored its members' previous activities on social networks (Twitter and Gitter) before it emerged into a GitHub-hosted project. Our finding emphasised that governance of social production needs to be investigated as an evolving phenomenon [1]. Our findings in the embryonic stage showed that governance was seen as the solution to social dilemmas. Therefore, the purpose of governance at the embryonic stage was to enhance connectivity and sociability between GitPoint members, while the purpose of governance changed in the emergence stage, and it was more concerned with how to manage operational software development processes (e.g. managing code quality). The established stage represents the maturity of GitPoint's repository, wherein governance was fully developed. In this stage, governance seems to have become increasingly formalised and embedded within the internal governance of GitHub's platform. In summary, previous literature suggests three different purposes for governance: "solving collective action problems, solving coordination problems in software 
development, and creating a better climate for contributors"; however, these purposes can be addressed simultaneously [30:157]. Therefore, in the established stage, the purpose of governance was not only to create a better climate for newcomers and incumbent users but also to solve social and coordination issues. Finally, we agree with the view of the purpose of governance that says it "is more compatible with a multidimensional perspective on OSS governance than with a monolithic perspective" [30:157].

Regarding the theoretical implications of our findings, we argue that in the existing literature, scholars studying governance in OSS communities, Wikipedia and production-based communities either focus on a dynamic process-oriented view or on static comparative analysis [3, 36], and they do so primarily in contexts where the online community is mature and well established. Also, the lack of an accepted definition leaves it up to each researcher to decide what governance means in communities. Thus, the conclusions based on the partially structural perspective used in prior studies do not reveal the full dynamics of governance typologies, configurations and elements that constitute governance in an online community.

The findings showed the importance of understanding the purposes of governance throughout the lifecycle of an online community and how these purposes change over time. Thus, managers who aim to set up online communities and enhance the activity and viability of a team might need to consider variations of governance mechanisms for better policy guidance. Pursuing this line of thought may identify situations where particular governance mechanisms are useful for one purpose but hurtful for another [30]. Yet, the present study has the following limitations: The suggested approach has been discussed only regarding its main aspect without going into much detail. We have explained the implications of our approach in the form of a theoretical narrative on the evolving governance of GitPoint. Further, GitPoint's members may have used other platforms for social networking that were not included in our data collection. Nevertheless, we are confident that our empirical data allowed us to capture what happened before, during and after the existence of GitPoint. Further research is needed to investigate the relationship between governance mechanisms and purposes and whether the presence and absence of specific governance mechanisms are consequential for project effectiveness.

\section{References}

[1] Aaltonen, A., and G.F. Lanzara, "Governing social production in the internet: The case of wikipedia", The Proceedings of 19th European Conference on Information Systems (ECIS), (2011).

[2] Aaltonen, A., and G.F. Lanzara, "Governing complex social production in the internet: The emergence of a collective capability in Wikipedia", SSRN Electronic Journal, 2011.

[3] Aaltonen, A., and G.F. Lanzara, "Building governance capability in online social production: Insights from

Wikipedia”, Organization Studies 36(12), 2015, pp. 16491673.

[4] Argyres, N.S., T. Felin, N. Foss, and T. Zenger, "Organizational economics of capability and heterogeneity", Organization Science 23(5), 2012, pp. 1213-1226.

[5] Bartlett, C., and S. Ghoshal, "Beyond strategy, structure, systems to purpose, process, people", MONASH MT ELIZA BUSINESS REVIEW 1, 1994, pp. 54-61.

[6] Benkler, Y., The wealth of networks: How social production transforms markets and freedom, Yale University Press, 2006.

[7] Benkler, Y., "Peer production, the commons, and the future of the firm", Strategic Organization 15(2), 2017, pp. 264-274.

[8] Berente, N., S. Seidel, and H. Safadi, "Research Commentary-Data-Driven Computationally Intensive Theory Development", Information Systems Research 30(1), 2018, pp. 50-64.

[9] Black, L.W., H.T. Welser, D. Cosley, and J.M. DeGroot, "Self-governance through group discussion in wikipedia: Measuring deliberation in online groups", Small Group Research 42(5), 2011, pp. 595-634.

[10] Child, J., and S.B. Rodrigues, "Corporate governance and new organizational forms: Issues of double and multiple agency", Journal of Management and Governance 7(4), 2003, pp. 337-360.

[11] Cobb, N.K., A.L. Graham, and D.B. Abrams, "Social network structure of a large online community for smoking cessation", American Journal of Public Health 100(7), 2010, pp. $1282-1289$.

[12] Dahlander, L., L. Frederiksen, and F. Rullani, "Online communities and open innovation", Industry and innovation 15(2), 2008, pp. 115-123.

[13] Dosi, G., M. Faillo, and L. Marengo, “Organizational capabilities, patterns of knowledge accumulation and governance structures in business firms: an introduction", 2008.

[14] Faraj, S., S.L. Jarvenpaa, and A. Majchrzak, "Knowledge collaboration in online communities", Organization Science 22(5), 2011, pp. 1224-1239.

[15] Faraj, S., G. von Krogh, E. Monteiro, and K.R. Lakhani, "Online community as space for knowledge flows", Information Systems Research 27(4), 2016, pp. 668-684. [16] Grant, R.M., "Prospering in dynamically-competitive environments: Organizational capability as knowledge integration", Organization science 7(4), 1996, pp. 375-387. [17] Harysi, M.A., B.; Negoita, and J. Nandhakumar, "The evolution of leadership structures in online communities: A social network perspective", Evolution, (2019). 
[18] Hayek, F.A., "The use of knowledge in society", The American economic review 35(4), 1945, pp. 519-530. [19] Hippel, E. von, and G. von Krogh, "Open source software and the 'private-collective' innovation model: Issues for organization science", Organization science 14(2), 2003, pp. 209-223.

[20] Jacobides, M.G., and S.G. Winter, "Capabilities: Structure, agency, and evolution”, Organization Science 23(5), 2012, pp. 1365-1381.

[21] Jensen, C., and W. Scacchi, "Collaboration, Leadership, Control-, and Conflict Negotiation in the Netbeans. org Community", 26th International Software Engineering Conference, IET (2004).

[22] Johnson, S.L., H. Safadi, and S. Faraj, "The emergence of online community leadership", Information Systems Research 26(1), 2015, pp. 165-187.

[23] Kim, A.J., Community building on the web: Secret strategies for successful online communities, AddisonWesley Longman Publishing Co., Inc., 2000.

[24] Kolbjørnsrud, V., "Agency problems and governance mechanisms in collaborative communities", Strategic Organization 15(2), 2017, pp. 141-173.

[25] Langley, A., "Strategies for theorizing from process Data”, Academy of Management Review 24(4), 1999, pp. 691-710.

[26] Lasfer, A., and E. Vaast, "A relational approach on collaborative resource spending in online communities", International Conference on Information Systems 2018, ICIS 2018, (2018), 1-17.

[27] Lindberg, A., N. Berente, J. Gaskin, and K. Lyytinen, "Coordinating interdependencies in online communities: A study of an open source software project", Information Systems Research 27(4), 2016, pp. 751-772.

[28] Lynn Jr, L.E., C.J. Heinrich, and C.J. Hill, Improving governance: A new logic for empirical research, Georgetown University Press, 2001.

[29] Madhok, A., "Reassessing the fundamentals and beyond: Ronald Coase, the transaction cost and resource- based theories of the firm and the institutional structure of production", Strategic Management Journal 23(6), 2002, pp. 535-550.

[30] Markus, M.L., "The governance of free/open source software projects: monolithic, multidimensional, or configurational?", Journal of Management \& Governance 11(2), 2007, pp. 151-163.

[31] Mockus, A., R.T. Fielding, and J.D. Herbsleb, "Two case studies of open source software development: Apache and Mozilla", ACM Transactions on Software Engineering and Methodology (TOSEM) 11(3), 2002, pp. 309-346.

[32] O'Mahony, S., "The governance of open source initiatives: What does it mean to be community managed?", Journal of Management and Governance 11(2), 2007, pp. 139-150.

[33] O’Mahony, S., and F. Ferraro, "The emergence of governance in an open source community", Academy of Management Journal 50(5), 2007, pp. 1079-1106.

[34] Preece, J., Online communities: Designing usability and supporting socialbilty, John Wiley \& Sons, Inc., 2000.

[35] Shah, S.K., "Motivation, governance, and the viability of hybrid forms in open source software development", Management science 52(7), 2006, pp. 1000-1014.

[36] Shaikh, M., and T. Cornford, "Version management tools: CVS to BK in the Linux kernel", 3rd Workshop on Open Source Software Engineering, (2003), 127-132. [37] Shaikh, M., and O. Henfridsson, "Governing open source software through coordination processes", Information and Organization 27(2), 2017, pp. 116-135. [38] Vaast, E., H. Safadi, L. Lapointe, and B. Negoita, "Social Media Affordances for Connective Action: An Examination of Microblogging Use During the Gulf of Mexico Oil Spill”, MIS Quarterly 41(4), 2017, pp. 1179 1205.

[39] Winter, S.G., "Understanding dynamic capabilities", Strategic management journal 24(10), 2003, pp. 991-995. [40] Zollo, M., and S.G. Winter, "Deliberate learning and the evolution of dynamic capabilities", Organization science 13(3), 2002, pp. 339-351. 